\title{
Quelles voies pour la participation du public à la conservation de la nature ? Le cas des réserves de biosphère
}

What pathways for public participation in nature conservancy? The case of the biosphere reserves

Jean-Eudes Beuret

\section{OpenEdition}

Journals

Édition électronique

URL : http://journals.openedition.org/developpementdurable/9096

DOI : 10.4000/developpementdurable.9096

ISSN : 1772-9971

Éditeur

Association DD\&T

Référence électronique

Jean-Eudes Beuret, "Quelles voies pour la participation du public à la conservation de la nature? Le cas des réserves de biosphère », Développement durable et territoires [En ligne], Vol. 2, $n^{\circ} 3$ | Décembre 2011, mis en ligne le 03 décembre 2011, consulté le 19 avril 2019. URL : http://

journals.openedition.org/developpementdurable/9096; DOI : 10.4000/developpementdurable.9096

Ce document a été généré automatiquement le 19 avril 2019

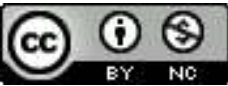

Développement Durable et Territoires est mis à disposition selon les termes de la licence Creative Commons Attribution - Pas d'Utilisation Commerciale 4.0 International. 


\title{
Quelles voies pour la participation du public à la conservation de la nature ? Le cas des réserves de biosphère
}

\author{
What pathways for public participation in nature conservancy? The case of the \\ biosphere reserves
}

Jean-Eudes Beuret

Les politiques de l'environnement ont, depuis longtemps, inscrit la participation du public à l'ordre du jour. En France, après s'être préoccupé du droit à l'information des citoyens, l'Etat met en place des procédures de consultation avec la loi Bouchardeau de 1983. Il se penche ensuite sur la participation du public, avec la circulaire Bianco de 1992 relative à la conduite des grands projets d'infrastructure, puis la loi Barnier de 1995, qui crée notamment la Commission nationale du débat public. À l'échelle internationale, dès 1992, le principe $n^{\circ} 10$ de la déclaration de Rio pose que «la meilleure façon de traiter les questions d'environnement est d'assurer la participation de tous les citoyens, à un niveau qui convient ». Si certains auteurs interrogent aujourd'hui ce postulat (La Branche, 2009, Raymond, 2009, Ballan, 2011), la participation reste généralement considérée comme partie intégrante du développement durable. Comment la mettre en place? Pour nombre de décideurs, la participation du public à la conservation de la nature évoque d'abord des actions de formation et d'information visant à faire évoluer les comportements, ou des instances de dialogue mises en place à grands renforts de réunions. Dans les territoires et aires protégées, ces instances prennent la forme de comités de pilotage et des procédures à caractère consultatif ou décisionnel sont mises en place afin d'élaborer des documents d'objectifs ou plans de gestion. Mais des travaux tels que ceux de Pretty $(1994,1995)$, montrent que le champ des possibles est beaucoup plus large et va d'une participation passive à l'auto-mobilisation du public. La diversité des voies et moyens de la participation du public reste à explorer. 
2 Nous nous sommes employés à le faire, à partir d'une analyse comparative menée dans dix pays ${ }^{1}$. Afin d'étudier des situations comparables, notre analyse a porté sur un même dispositif de conservation de la nature, les « réserves de biosphère ». Selon le programme Man And Biosphere de l'UNESCO, en cherchant à réconcilier le développement économique, le développement social et la conservation de la biodiversité, grâce à des alliances entre les populations locales et les milieux naturels, les réserves de biosphère constituent des lieux privilégiés pour expérimenter des pratiques de développement durable à une échelle territoriale. Mais comment mettre en place ces alliances? La voie du dialogue et de la participation du public est proposée par la « stratégie de Séville » énoncée en 1995 (Bouamrane, 2006), qui pose des principes directeurs pour les réserves de biosphère : elle évoque un "pacte" à nouer entre la communauté locale et la société dans son ensemble ainsi que le fait de « recenser les intérêts des différents partenaires et intégrer pleinement ces différents acteurs sociaux aux processus de planification et de prise de décision ».

3 Comment ces principes de participation ont-ils été traduits en actions et dispositifs ? Nous nous sommes intéressés aux réponses apportées sur le terrain, dans des contextes économiques, politiques et culturels très divers (au Bénin, Brésil, Canada, Cambodge, Corée, France, Guatemala, Inde, Uruguay, Vietnam). Après avoir présenté notre démarche d'analyse, nous décrirons la gamme des solutions expérimentées pour assurer la participation du public, puis aborderons les questions-clés posées par la mise en dialogue de l'environnement et du développement.

\section{La démarche d'analyse}

\subsection{Terrains, collecte des données, échantillonnage}

La désignation « réserve de biosphère » est attribuée par l'Unesco à des territoires dans lesquels une politique de conservation et de développement durable est mise en place, à l'initiative du territoire concerné, de l'Etat, parfois des citoyens. Ce sont souvent des aires protégées préexistantes qui sollicitent cette désignation: l'Unesco anime le réseau constitué par ces réserves mais n'a aucun rôle dans leur gestion. Ce réseau s'est doté de principes communs: chaque réserve compte ainsi une ou plusieurs zones centrales soumise(s) à des mesures spécifiques de conservation de la biodiversité et une zone tampon dans laquelle il est surtout question de concilier le développement économique et la conservation de l'environnement. On y trouve des acteurs qui entendent développer leurs activités économiques, d'autres acteurs qui veulent conserver la biodiversité, une autorité de gestion qui dispose de prérogatives plus ou moins affirmées sur le territoire, un grand public plus ou moins au fait des objectifs et parfois même de l'existence de la réserve. Au-delà de principes communs et d'une même désignation, les 580 réserves de biosphère qui existent aujourd'hui sont extrêmement variées : certaines reposent sur un dispositif réglementaire très développé alors qu'aucune mesure contraignante n'existe dans d'autres cas ; certaines disposent de moyens importants alors que d'autres n'existent que sous la forme d'un plan supposé orienter les politiques locales; enfin, alors qu'elles sont généralement créées par les pouvoirs publics, ce sont des initiatives citoyennes dans le cas du Canada. Mais le fait d'y retrouver des principes et objectifs communs en fait un cadre pertinent pour une analyse comparative : elles permettent de voir comment, dans le même cadre organisationnel mais dans des contextes très différents, chacun tente de 
répondre à la question de la participation du public à la conservation de la nature. Nous avons sélectionné douze cas de façon à maximiser la diversité des enjeux en termes de développement et de conservation, ainsi que la diversité culturelle, politique et économique (Tableau 1).

TABLEAU 1. L'ÉCHANTILLON ÉTUDIÉ : OPTIMISER LA DIVERSITÉ DES SITUATIONS

\begin{tabular}{|l|l|l|}
\hline Pays & Réserve de biosphère & Enjeux en termes de biodiversité \\
\hline Bénin & Pendjari & Forêt, faune. Développement local dans la zone tampon \\
\hline Inde & Nanda Devi & Flore de haute montagne, développement local \\
\hline Cambetnam & Can Gio Mangrove & Mangrove \\
\hline Cambodge & Koh Kong (projet) & Oiseaux, ressources halieutiques \\
\hline Corée du Sud & Jeju Island & Ressources halieutiques, forestière. Tourisme \\
\hline Guatemala & Maya & Forêt. Problèmes d'installations illégales \\
\hline Uruguay & Banãdos del Este & Conservation de zones humides \\
\hline Brésil & SaoPaolo/ceinture verte & Forêt péri-urbaine menacée par des infrastructures \\
\hline Canada & Lac Saint Pierre & Zones humides, faune et flore \\
\hline Canada & Manicouagan & Forêt. Exploitation minière, forestière, barrages \\
\hline France & Vosges du Nord & Faune et flore. Réserve transfrontalière \\
\hline
\end{tabular}

Cet échantillon n'est pas représentatif et ne permet pas de couvrir la diversité des situations existantes, mais il est suffisamment restreint pour réaliser une analyse approfondie de chaque cas, tout en permettant d'approcher une certaine diversité de situations.

Chacune des réserves a fait l'objet d'une analyse monographique, basée sur l'analyse de la documentation existante, des visites de terrain, des entretiens semi-directifs auprès des principaux acteurs de la réserve et, lorsque cela a été possible, l'observation de temps de dialogue. Est considérée comme acteur de la réserve toute personne dont certains actes affectent le territoire de cette réserve, en tant qu'usager de ressources ou d'espaces qu'elle inclue, en tant que porteur d'une demande relative à certains des biens d'environnement qu'elle supporte, ou en tant qu'intervenant institutionnel. Ces personnes sont en interaction au sein d'un « jeu d'acteurs » : afin de représenter ce jeu, est considéré comme une catégorie d'acteurs un groupe composé de personnes physiques ou morales caractérisées par les mêmes types d'actes relatifs à la réserve et les mêmes comportements et prises de positions dans le jeu des acteurs. Deux personnes ou groupe sociaux qui exercent la même activité mais ne défendent pas la même position, en raison d'intérêts, de perceptions ou d'opinions différentes, relèvent de catégories distinctes. Dans un premier temps, des entretiens auprès de grands témoins de l'histoire du territoire et l'analyse de la bibliographie existante nous ont permis à la fois d'approcher le territoire, de distinguer des catégories d'acteurs et de constituer un échantillon de personnes à rencontrer. Les entretiens ont été réalisés par la même personne, sur la base d'une grille d'analyse préétablie, commune à toutes les réserves, afin de faciliter les comparaisons. Dans chaque cas, les entretiens visaient à identifier et analyser d'une part les moyens (règles, organisations, instances...) grâce auxquels le concept de développement durable trouve une application concrète dans ces réserves, d'autre part les formes de participation du public et de facilitation du dialogue entre les acteurs. Dans 
la plupart des cas, nous avons pu en outre assister à des temps de dialogue entre l'autorité de gestion de la réserve et des communautés locales, voire à des temps de négociation entre cette autorité et des groupes porteurs d'un intérêt spécifique (dans les cas du Nanda Devi et du Tonle Sap).

Cette analyse reste limitée par le temps passé dans chaque réserve (une à deux semaines), mais surtout par la position de l'enquêteur, mandaté par l'Unesco auprès de l'autorité de gestion de la réserve pour réaliser cette recherche, ce qui affectait d'emblée son indépendance. Dans certaines réserves de biosphère, l'autorité de gestion n'a joué qu'un rôle de facilitation pour l'accès aux données et aux acteurs, avec peu d'interférences, mais le fait d'afficher un intérêt pour les formes de participation mises en place créait de toute façon un biais dont il nous a fallu tenir compte, certains acteurs opposés à toute participation ne souhaitant pas dévoiler leur point de vue. Malgré ces limites, l'approche multi-sites, comparative, nous semble pertinente : elle permet d'établir en quoi certains facteurs locaux déterminent les dispositifs de participation retenus mais surtout, faisant l'hypothèse que ces dispositifs ne sont pas entièrement déterminés par le contexte de leur mise en œuvre, de révéler des récurrences qui ressortent d'autant plus clairement que les situations sont diverses. Retrouver par exemple des mécanismes basés sur les mêmes principes au Vietnam et au Guatemala permet d'en saisir le sens, d'autant plus que les contextes culturels, économiques et politiques sont radicalement différents.

\subsection{La participation du public à la conservation de la nature : définitions}

8 La participation du public à la conservation de la nature peut emprunter deux voies. Soit des individus ou groupes sociaux prennent l'initiative d'agir en faveur de la conservation de la nature (Beuret, Cadoret, 2010), participant ainsi à une action publique définie par Trom (2003) comme toute activité articulée sur un espace public et nécessitant une référence à un bien commun, définition extensive qui permet de réunir dans un même cadre des activités directement liées à la puissance publique et celles issues d'initiatives citoyennes. Soit ces individus ou groupes sociaux entendent participer à une politique publique de gestion de la nature : la participation du public recouvre alors tous les actes par lesquels le public, invité ou non à le faire, tente d'agir pour influencer les objectifs de cette politique (et parfois son existence) ou contribuer à ce qu'ils soient atteints.

Nous intéressant à la participation du public dans le cas des réserves de biosphère, nous considérerons comme étant «le public» tous les acteurs de la réserve tels que définis précédemment, à l'exception de l'autorité de gestion de la réserve et des organisations qui lui sont liées par des relations hiérarchiques. Ils peuvent investir un espace public dans lequel ils peuvent s'exprimer et/ou agir pour tenter d'influencer l'action de la réserve, ou être invités à le faire par l'autorité de gestion de la réserve, désireuse de stimuler leur participation. Le concept d'espace public, défini par Habermas (1978), repose sur la liberté et l'autonomie des citoyens pour la formation par la raison d'une opinion et d'une volonté collective qui viendraient influer sur la production de lois. Il s'agit ici d'influencer la gestion et le devenir de la réserve. Cet espace d'expression et d'action peut être indépendant de l'autorité de gestion de la réserve, ou être organisé par cette dernière. Dans l'espace public, les acteurs peuvent se retrouver dans des espaces de dialogue, assimilables à des forums hybrides locaux (Callon et al. 2001). Ils sont qualifiés de forums parce qu'il s'agit d'espaces ouverts où les groupes peuvent se mobiliser pour 
débattre de choix qui engagent le collectif et d'hybrides «parce que ces groupes engagés et les porte-parole qui prétendent les représenter sont hétérogènes : on y trouve à la fois des experts, des hommes politiques, des techniciens et des profanes qui s'estiment concernés. (Callon et al., 2001). Leur caractère hybride vient aussi des thèmes de discussion qui associent des questions économiques, écologiques, sociales.

La participation du public s'inscrit dans une gouvernance que nombre de décideurs appellent de leurs vœux. Selon Bertrand et Moquay (2004), la gouvernance recouvre tout système de décision publique intégrant des acteurs privés. Au-delà de cette dichotomie, Jean et Ependa Muteba Wa (2004) distinguent trois grands ordres de pouvoir ou forces sociales qui doivent être partie prenante de la gouvernance : le pouvoir politique, c'est-àdire le gouvernement local; le pouvoir économique qui comprend les entreprises du secteur privé ; le pouvoir social des citoyens réunis dans différents groupes d'intérêts et des associations volontaires, ce que l'on appelle aussi la « société civile ». L'enjeu majeur de la gouvernance est non seulement de dépasser les frontières entre ces forces sociales, mais aussi de dépasser les frontières existant au sein de chacune d'entre elles. Dans les réserves de biosphère, ces frontières se manifestent notamment par une gestion en mosaïque (Beuret, 2006c), diverses organisations publiques ou privées disposant de prérogatives distinctes sur différents objets ou fractions du territoire de la réserve. Chacune dispose de prérogatives, sur cet objet et/ou dans cet espace, qu'elle n'a pas ailleurs et que les autres n'ont pas : le public étant confronté à toutes ces organisations, la participation du public peut aider à dépasser ces cloisonnements. De façon générale, à l'instar de Laganier, Villalba et Zuindeau (2002), nous envisagerons ici la gouvernance comme un mode d'organisation des acteurs (institutions, entreprises, associations) plus fondé sur l'accommodement que sur la hiérarchie ou la domination, destiné à concevoir et à mettre en œuvre un projet. Le projet est ici la réserve de biosphère dans ce qu'elle est et dans ce qu'elle fait: la participation du public est une composante essentielle de sa gouvernance.

\section{Les figures de la participation du public}

\subsection{Plusieurs niveaux, plusieurs voies}

11 Parmi les nombreuses typologies existantes des formes de participation du public (Beierle, 1999; Fung, 2003; Rowe et Frewer, 2005; Fung, 2006), nous en avons retenu deux, certes plus anciennes mais qui se révèlent éclairantes au vu des situations de terrain étudiées et adaptées à l'analyse de dynamiques territoriales. La typologie d'Arnstein (1969) reste une référence et explicite certaines composantes des formes de participation identifiées, en insistant sur la répartition du pouvoir entre le décideur et les participants. Arnstein (1969) distingue d'abord deux formes (manipulation et thérapie) qui ne laissent pas de place à une réelle participation, où l'objectif du décideur est d'éduquer ou de "soigner » les participants, en vue de les convaincre de la pertinence de ses décisions. Nous retrouverons ces objectifs en arrière-plan de certaines formes d'information et de consultation. Il distingue ensuite des opérations dans lesquelles le pouvoir de décision reste exclusivement détenu par celui qui propose aux citoyens de participer, même s'il invite ces derniers à s'exprimer pour fournir des informations et des avis, voire à formuler certains conseils. Ce sont des opérations d'information, de consultation puis d'apaisement, ces dernières étant marquées par une plus grande ouverture du décideur 
aux avis et conseils qui sont énoncés par les participants, même s'il s'agit d'un geste symbolique qui ne remet pas en cause l'absence de partage du pouvoir de décision : là encore, nous retrouverons ceci en arrière-plan de certaines formes de participation identifiées. Viennent ensuite trois niveaux qui correspondent à l'octroi d'un pouvoir de décision plus ou moins important aux citoyens: le partenariat, avec un partage de responsabilités au sein de comités, la délégation de pouvoir avec des comités à caractère décisionnel dominés par les citoyens, puis le contrôle par les citoyens, ceux-ci assumant la prise de décision tout en ayant un accès direct aux sources de financement.

12 En réalité, la typologie d'Arnstein nous semble limitée par la question centrale qu'il se pose, qui est celle du partage du pouvoir. A titre d'exemple, dans les formes de participation que nous avons identifiées, la construction collective du projet par la concertation peut se faire avec ou sans partage du pouvoir de décision final: la participation est alors déterminante et la répartition du pouvoir est une question secondaire. Dépasser cette question permet d'approcher d'autres dimensions de la diversité des situations, révélées en particulier par Pretty (1995). Les cas de figures observés dans les réserves de biosphère s'inscrivent globalement dans la typologie proposée par cet auteur, avec certaines différences (Tableau 2).

Si le premier niveau distingué par Pretty (1994) est la participation passive, il nous faut distinguer un niveau zéro de la participation qui correspond à l'exclusion du public. L'exclusion de toute activité humaine dans certaines zones induit une absence totale de participation et des effets négatifs sur lesquels nous reviendrons. Pretty (1994) distingue ensuite la " participation passive », la « participation par collecte d'informations » et la " participation par consultation »: dans les réserves de biosphère, on observe en réalité un continuum entre de véritables consultations, des pratiques de consultation qui n'ont d'autre effet que l'information du public, des pratiques simples d'information et des pratiques visant plus à convaincre qu'à informer, où l'on retrouve les objectifs des opérations qualifiés de manipulation et de thérapie par Arnstein (1969). Nous qualifions le niveau suivant de " participation contributive » : le public apporte une contribution à un projet décidé et piloté par d'autres, au nom de la protection de la nature, ce qui rejoint la "participation en échange d'incitations matérielles» ou la "participation fonctionnelle ». La participation reste limitée car elle est instrumentalisée et le public n'a d'autre choix que d'accepter ou de refuser une collaboration, voire un contrat. La concertation correspond à la "participation interactive", à ceci près que ce qui caractérise la concertation est l'existence d'une construction collective mais qu'elle ne va pas toujours jusqu'à la conception commune de l'ensemble du projet. Enfin, nous avons observé un certain nombre de cas où l'autorité de gestion de la réserve de biosphère s'emploie à stimuler l'auto-mobilisation, puis à la faciliter. Dans d'autres cas, elle émerge d'elle-même. 
Tableau 2. la participation dans les réserves de biosphère, au regard des niveaux de participation proposés par Pretty (1994)

\begin{tabular}{|c|c|}
\hline Typologie de Jules Pretty (1994) & $\begin{array}{l}\text { Typologie proposée à partir d'une } \\
\text { mise en perspective du cas des } \\
\text { réserves de biosphère }\end{array}$ \\
\hline & L'exclusion \\
\hline $\begin{array}{l}\text { La participation passive : le public est informé de } \\
\text { ce qui va se passer }\end{array}$ & \multirow{3}{*}{$\begin{array}{l}\text { L'information } \\
\text { et la consultation }\end{array}$} \\
\hline $\begin{array}{l}\text { La participation par la collecte d'informations : le } \\
\text { public répond à des questions prédéfinies }\end{array}$ & \\
\hline $\begin{array}{l}\text { La participation par consultation : le public donne } \\
\text { son propre avis }\end{array}$ & \\
\hline $\begin{array}{l}\text { La participation en échange d'incitations } \\
\text { matérielles : le public participe en échange d'un } \\
\text { accès à certaines ressources }\end{array}$ & \multirow{2}{*}{ La participation contributive } \\
\hline $\begin{array}{l}\text { La participation fonctionnelle : la participation du } \\
\text { public crée des conditions favorables pour la } \\
\text { réussite du projet }\end{array}$ & \\
\hline $\begin{array}{l}\text { La participation interactive : le public participe à } \\
\text { l'analyse conjointe et décide de la suite du projet. }\end{array}$ & La concertation \\
\hline $\begin{array}{l}\text { L'auto-mobilisation : le public prend ses propres } \\
\text { initiatives }\end{array}$ & $\begin{array}{l}\text { L'auto-mobilisation stimulée par la } \\
\text { réserve : elle crée un milieu innovant et } \\
\text { actif en faveur de la nature }\end{array}$ \\
\hline
\end{tabular}

\subsection{L'exclusion et ses écueils}

L'exclusion des usages anthropiques dans certaines zones centrales interdit toute participation dans ces zones et pose des problèmes tant pour l'homme que la nature. Non seulement les expulsés perdent l'accès à certaines ressources mais ils subissent des effets externes de la zone d'exclusion vers la zone périphérique : en Inde comme au Bénin, les habitants des zones périphériques des zones d'exclusion subissent des pertes de cultures dues à la faune sauvage. Pour la nature, les effets négatifs sont plus nombreux qu'il n'y parait, d'abord dans ces zones périphériques, du fait des déséquilibres induits par ces changements dans les systèmes de production. En Inde, l'interdiction des activités humaines dans une zone centrale de la réserve du Nanda Devi a induit une réduction du bétail en raison de la raréfaction des zones de pâturage. Il en résulte une diminution des apports de matière organique, avec une baisse de la fertilité des sols et de sérieux problèmes d'érosion. Par ailleurs, l'interdiction des expéditions en montagne signifie le tarissement d'une source de revenus monétaires : les familles ont réagi en augmentant la part de cultures de vente, avec une réduction de la biodiversité liée aux cultures traditionnelles. De façon générale, on assiste à un report de la pression humaine sur la zone périphérique: non seulement cette zone concentre toutes les frustrations de ceux qui ont perdu l'accès à des ressources ou à des éléments essentiels de leur culture, mais on crée une zone très dégradée du point de vue environnemental. Au sein même de la zone d'exclusion sont parfois observés des effets négatifs, avec la rupture d'équilibres écologiques maintenus par des activités humaines: sur l'île de Jeju, en Corée du Sud, l'interdiction du pâturage dans la zone centrale a eu pour conséquence l'invasion des étages écologiques supérieurs par une plante indésirable. Ces cas révèlent le fait que même lorsque le public ne participe pas, il contribue parfois par ses activités au maintien 
de certaines dynamiques naturelles : on aurait tort de se priver de cette participation contributive implicite.

\subsection{L'information et la consultation : un continuum de pratiques}

15 La participation du public est basée le plus souvent sur des mécanismes d'information et de consultation. Elle a lieu lors de la création de la réserve et/ou après, autour de problèmes spécifiques. Nous avons observé un continuum de pratiques avec quatre niveaux de référence : des pratiques limitées à l'information du public; des consultations informatives où l'échange d'informations est la fonction centrale; des consultations contributives dont la fonction centrale est la collecte d'avis et de propositions; des consultations interactives qui favorisent des échanges entre les participants d'une part, entre les consultés et celui qui consulte d'autre part.

Certes, l'information du public est primordiale pour lui permettre de prendre la parole, d'investir l'espace public et revient à lui donner du pouvoir (Atger et al., 2000). Mais lorsque l'information reste l'objectif central des opérations menées, elle vise souvent plus à satisfaire une obligation qu'à stimuler la participation et à réduire de façon effective les asymétries informationnelles existantes. La consultation peut aller plus loin en termes de participation, mais ses modalités sont très variables et plusieurs paramètres déterminent sa portée. C'est d'abord le temps que l'on se donne. La consultation est souvent trop ponctuelle pour que les participants s'approprient les questions posées et l'information nécessaire à une prise de position. A l'inverse, certaines réserves mettent en place des instances où les mêmes participants avancent ensemble dans un apprentissage collectif. C'est ensuite les conditions de l'ouverture de la consultation, en amont ou en aval de travaux techniques. Consulter le public au sujet de la réserve ou d'un espace dont le plan de gestion a déjà été élaboré pose problème. Le niveau d'engagement quant à la prise en compte des idées exprimées joue ensuite un rôle clé : il n'est jamais fixé de façon explicite mais d'une part les consultés gardent en mémoire leurs expériences passées, d'autre part il semble exister des références conventionnelles tacites (Favereau, 1989). Dans certains pays, ceux qui consultent affirment avoir l'obligation de tenir compte des idées exprimées, d'une façon ou d'une autre, en l'absence de toute règle explicitement posée. Ceci est très déterminant pour la qualité de la participation. D'autres éléments liés à la conduite de cette opération jouent un rôle, tels que la clarté de la question posée ou la latitude offerte aux participants dans la définition des questions ; les moyens investis, qui permettent de limiter la taille et l'hétérogénéité des groupes consultés; la qualité et le caractère facilement appropriable des informations fournies.

\subsection{Une participation contributive, contractuelle}

Dans certains cas, le public est invité à apporter sa contribution à la conservation, en y trouvant son intérêt, dans le cadre de dispositifs contractuels de participation contributive. Deux portes d'entrée sont possibles. C'est d'abord la conservation, avec des acteurs rémunérés pour y participer et qui, en contrepartie, sont autorisés à réaliser certaines activités d'extraction. C'est le cas des 160 «familles protectrices» de la mangrove de Can Gio, au Vietnam. Le gouvernement leur a proposé de s'installer dans la forêt en leur offrant l'accès à des terres, ainsi qu'une subvention faible mais régulière : elles s'engagent en retour à protéger, gérer et utiliser la forêt en suivant les règles fixées 
par l'autorité de gestion de la réserve. C'est toute la famille qui participe à la surveillance d'environ cent hectares, souvent à l'occasion d'activités qui ont d'autres objectifs. Une activité économique constitue une seconde porte d'entrée possible, avec des acteurs bénéficiaires de concessions, qui y mènent une activité d'exploitation très encadrée par des règles qui visent la reproduction des ressources, tout en contribuant à la conservation. C'est le cas dans la réserve Maya, au Guatemala, dont la zone tampon est gérée via l'octroi de concessions forestières (de 20000 à 85000 hectares) à 14 communautés et 2 entreprises. Outre l'obligation de surveiller la concession, le concessionnaire a de nombreuses obligations relatives à l'activité d'extraction : il doit obtenir une certification forestière accordée par un organisme international indépendant et les cycles de coupe sont supervisés par l'autorité de gestion de la réserve.

La participation est ici très limitée car les "protecteurs » ou " concessionnaires » n'ont aucun droit de regard sur l'action d'ensemble de la réserve: ils apportent une contribution et leur autonomie de décision est limitée par le strict cadre du contrat qui leur est proposé. Mais ces mécanismes se révèlent efficaces à la fois en termes de conservation et de développement : d'une part ils permettent l'exploitation raisonnée de certaines ressources, d'autre part on constate que des espaces vides d'activités humaines, directement gérés par les pouvoirs publics, sont beaucoup plus sujets aux installations et activités illégales que les espaces qui sont gérés par une communauté locale. Dans le cas de la réserve Maya, la comparaison par photos aériennes de la couverture forestière d'espaces gérés par concession et d'espaces théoriquement interdits d'accès, montre que ces derniers sont beaucoup plus dégradés.

\subsection{Lorsque participation rime avec concertation et légitimation}

Dans d'autres cas, ce sont des processus de concertation qui supportent la participation. La concertation repose sur une volonté de coopération (Touzard, 2006), pour la construction collective de visions, d'objectifs, de projets communs, en vue d'agir ou de décider ensemble (Beuret, 2006a). Des processus de ce type ont été observés soit pour construire la réserve de biosphère, ce qu'elle sera et ce qu'elle fera, soit pour rendre un avis aux pouvoirs publics sur une question qui divise les acteurs, en réponse à une consultation. Par exemple à Koh Kong, au Cambodge, une ONG engage en 1997 un processus de recherche participative, avec les villageois, dont le but est de concevoir une gestion communautaire des ressources côtières. Les premières années permettent de créer un climat de confiance nécessaire à l'expression de tous, de collecter des informations en valorisant les savoirs locaux, de faire prendre conscience aux villageois du lien entre la conservation des ressources et les opportunités économiques dont ils disposent, pour les intéresser à cette question. Sur cette base sont créés des Comités villageois de gestion qui sont des lieux de concertation et d'action collective: les villageois engagent par eux-mêmes des actions avec l'appui de l'équipe de recherche participative, qui agit comme facilitatrice. Sur cette base est envisagée la création d'une réserve de biosphère, avec un projet co-construit par tous via des ateliers auxquels participent l'ensemble des groupes d'intérêt et des intervenants institutionnels. Parfois, l'autorité de gestion conduit une concertation via l'organisation de forums des acteurs de la réserve et/ou au sein d'un comité de gestion de la réserve. Dans la réserve de la ceinture verte de Sao Paolo, au Brésil, le comité de gestion est mobilisé pour produire le plan d'action de la réserve: il a également été sollicité pour rendre un avis sur une 
question qui divise ses participants, relative à la construction d'une autoroute. La concertation a permis de construire un avis unique, qui a d'autant plus de poids qu'il est porté par tous les acteurs.

La voie de la concertation semble présenter certains avantages : co-construire un avis ou un projet suppose le partage et l'appropriation réelle d'informations, avec une réduction substantielle des asymétries d'informations entre participants. Elle permet surtout de rendre plus légitime le produit de la concertation, qu'il s'agisse d'un avis, du plan de gestion de la réserve, ou de la réserve elle-même. Certaines réserves reposent certes sur un arsenal réglementaire conséquent mais ni ceci, ni la désignation accordée par l'Unesco ne suffit à les rendre légitimes aux yeux des acteurs locaux: la concertation permet de construire ou de consolider cette légitimité.

\subsection{Créer un milieu dialoguant et actif en faveur de la nature}

21 Dans les cas précédents, l'autorité de gestion rassemble les acteurs autour de son projet, de façon centripète, mais elle peut aussi choisir de faire émerger des actions en faveur de la nature, à partir de son projet mais de façon décentralisée et centrifuge. Il s'agit d'assurer la participation du public à un ensemble d'actions visant à atteindre les objectifs de la réserve, mais portées par le public lui-même. C'est le choix du " lâcher prise » : ceci suppose de renoncer à centraliser les actions pour accompagner des initiatives qui échapperont au contrôle de la réserve mais doivent contribuer à ce qu'elle atteigne ses objectifs.

Nous avons observé ceci par exemple dans la réserve de biosphère de la ceinture verte de Sao Paolo. Sa structure de gestion est très réduite mais elle a mis en place des centres d'éco-formation en partenariat avec des organisations disséminées dans le territoire. Des jeunes issus de quartiers défavorisés y suivent un cycle de formation de deux ans sur l'éco-tourisme, l'éducation à l'environnement, l'agriculture écologique, les pépinières forestières, la gestion des déchets, etc., suivi d'une mise en pratique à travers des projets. Ce sont eux qui devront engager des actions de conservation de la nature : il ne s'agit donc pas seulement de leur transmettre des connaissances mais de développer leur capacité entrepreneuriale en faveur du développement durable. Afin que ces compétences soient mobilisées, la réserve accompagne les jeunes dans l'après-formation, tente de stimuler le marché des "éco-jobs " et a mis en place une pépinière d'écoentreprises.

D'autres réserves mettent à disposition du public des points de repère et supports de compromis entre conservation et développement, dont il peut se saisir. Ce sont par exemple des modèles technologiques potentiellement labelisables, optimisés à la fois en terme économique et de conservation de la biodiversité. Au Canada, la réserve de biosphère de Manicouagan a ainsi engagé avec un partenaire industriel la recherche de modes d'exploitation de la forêt visant à limiter les impacts négatifs du point de vue paysager : le modèle mis au point pourra servir de référence pour d'autres entreprises.

24 La réserve de biosphère peut enfin faciliter l'émergence de scènes de concertation associant le public à l'échelle de la réserve, d'un espace, d'une ressource, ou d'un secteur d'activité. Au Canada, la réserve du lac Saint Pierre a favorisé l'émergence d'une table de concertation des élus du Lac Saint Pierre, ainsi que d'espaces de concertation décentralisés, autant d'instances indépendantes de la réserve. A Manicouagan, la réserve s'emploie à créer des espaces de concertation sectoriels, impliquant chacun des trois 
secteurs industriels (miniers, forestiers, de production d'hydro-électricité) qui modèlent le territoire. Ces deux réserves canadiennes s'emploient à créer un " milieu dialoguant ", autour et pour la conservation de la nature, ouvert à tous. Il s'agit en réalité de tenter d'optimiser un système de concertation constitué par les espaces de dialogue existant autour de la conservation de la nature. Il s'agit d'un système (De Rosnay, 1975) car d'une part ces espaces sont en interaction dynamique, notamment via des acteurs présents sur plusieurs scènes, d'autre part ils ont des objectif commun liés à la conservation de la nature. La réserve peut renforcer ce système en favorisant un engagement explicite sur des objectifs de développement durable: la réserve de Manicouagan envisage ainsi de réunir les acteurs de ces espaces autour d'une «charte d'engagements éthiques pour le développement durable ». Elle peut renforcer les liens entre les espaces de dialogue : à Manicouagan, la réserve a réussi à nouer des liens avec le secteur privé tout en étant lié aux associations de protection de la nature issues du grand public. Elle peut enfin identifier et agir sur les lacunes du système : par exemple autour $\mathrm{du}$ lac Saint Pierre, la réserve de biosphère a constaté que les controverses environnementales avaient suscité la création de mécanismes de protection et d'instances de dialogue suffisantes mais qu'il manquait une concertation entre les acteurs de l'écotourisme. Elle s'est attachée à combler cette lacune qui nuisait à la cohérence du système, en créant une coopérative de solidarité centrée sur cette question, avec les acteurs concernés.

Dans tous ces cas, la réserve stimule la participation du public non plus autour de ses propres actions (qui sont alors très limitées), mais en stimulant l'émergence d'actions et d'espaces de dialogue décentralisés: ce sont des acteurs que la réserve dote de compétences et accompagne dans leurs initiatives, des espaces de dialogue dont elle appuie l'émergence ainsi que des liens qu'elle crée entre les espaces de dialogue existants, des références et modèles technologiques pertinents en termes de conservation dont elle appuie la mise au point et la diffusion. Il s'agit de créer un milieu dialoguant et actif en matière de conservation de la nature, en y disséminant des ressources et en favorisant des processus d'apprentissage. Cette logique se rapproche de celle des milieux innovateurs (Camagni, Maillat, 2006) qui met l'accent sur le rôle des processus d'apprentissage et d'interaction dans la création de ressources spécifiques à l'origine de l'innovation (Coppin, 2002), dans un milieu donné. C'est une voie qui reste peu explorée dans les travaux qui portent sur la participation du public à la conservation de la nature.

\section{Quels espaces de dialogue?}

Lorsque la participation du public passe par des processus d'échange d'information, de consultation, de concertation, de création d'un milieu dialoguant, des espaces de dialogue sont mis en place, avec là encore l'exploration de modalités très diverses.

\subsection{La création d'instances de dialogue : des choix qui ne sont pas neutres}

Nombre de réserves créent des instances de dialogue pour la participation du public. Elles le font en répondant de diverses façons aux trois questions suivantes, ce qui n'est pas anodin. 

associent les stakeholders: c'est l'activité, l'usage des ressources qui ouvre l'accès à l'expression et à la participation. Au Canada, le comité de gestion de la réserve de Manicouagan associe, outre les municipalités et quatre citoyens, les secteurs industriels de l'énergie, des mines, de la forêt, puis la protection de la faune, le tourisme, l'éducation, l'environnement, le milieu économique local. Dans d'autres cas, ce sont les habitants d'un territoire qui sont représentés. En Inde, la réserve de biosphère du Nanda Devi a mis en place des Comités d'Eco-Développement (CED) qui réunissent les villageois et l'autorité de gestion. Selon Maikhuri et Rao (2006), même si les espaces naturels gérés par la réserve appartiennent au gouvernement, ce dernier doit reconnaître des droits aux communautés, car ce sont elles qui ont protégé cette réserve depuis des centaines d'années. C'est alors l'appartenance locale qui ouvre l'accès à la participation, ce qui revient à reconnaitre des droits sur la nature à la communauté attachée à ce territoire.

La priorité est-elle accordée à la mobilisation ou à la représentativité ? Si l'échelle reste modeste, la participation peut être ouverte à tous : une sélection s'opère d'elle-même en fonction de la motivation et de la disponibilité de chacun, ce qui nuit à la représentativité des instances mais favorise la mobilisation. Face à ce biais, la réserve peut privilégier la représentativité en n'invitant que des représentants. Ceci pose des problèmes d'identification des catégories représentées, de choix des représentants qui doivent être légitimes tant pour ceux qu'ils représentent (légitimité interne) qu'aux yeux des autres acteurs du dialogue (légitimité externe), d'absence de représentation organisée de certains acteurs. Certaines réserves s'emploient à faciliter l'émergence d'organisations et de représentants légitimes, en amont de la constitution d'instances de participation. Ceci devient très compliqué lorsque le territoire est immense et qu'il s'agit d'une unité fonctionnelle irréductible : il faut alors envisager un travail très lourd de renforcement d'instances de représentation du public.

Quel degré d'autonomie est accordé à ces instances? Là encore, les choix ne sont pas neutres. Certaines sont créées comme des espaces d'auto-mobilisation: les comités villageois de Koh Kong au Cambodge ou la commission de la Laguna Rocha en Uruguay choisissent eux-mêmes leurs règles constitutives et de fonctionnement et construisent leurs actions, la réserve n'étant que facilitatrice. D'autres sont des lieux d'interface : les CED, en Inde, sont présidés par un villageois mais le secrétaire est un agent de la réserve et les deux signatures sont nécessaires pour engager des dépenses. D’autres sont de simples courroies de transmission de messages préfabriqués et s'inscrivent dans une participation passive au sens de Pretty (1994).

\subsection{Création, optimisation et réarrangements institutionnels}

31 La création institutionnelle ne doit pas se faire sans lien avec ce qui existe déjà et sans un effort d'optimisation de l'existant. C'est effectivement ce que l'on observe par exemple sur l'île de Jeju, en Corée, qui dispose d'outils de planification spatiale qui constituent une base solide, puis de mécanismes de régulation environnementale, avec la réalisation d'études d'impact et l'action d'associations de protection de la nature qui interpellent les autorités en cas de problèmes. Au vu de ces mécanismes, l'autorité de gestion de la réserve n'a pas créé de nouvelles instances de dialogue mais a tenté d'optimiser les coordinations existantes

Développement durable et territoires, Vol. 2, n 3 | Décembre 2011 
$32 \mathrm{Au}$ sein des instances mises en place, des réarrangements institutionnels négociés s'avèrent parfois très puissants. L'expérience des CED, en Inde, montre qu'ils peuvent suffire à créer des dynamiques locales de participation très fructueuses. A Bundyar et Govindghat, ces comités se sont vus affecter le produit de taxes auparavant prélevées par un district, à l'issue d'une négociation facilitée par la réserve. Ces villages voient passer un nombre très important de pèlerins, qui font vivre une économie locale mais laissent beaucoup de déchets. En contrepartie des taxes reçues, chaque CED s'est engagé à gérer le site, le nettoyer et reverser $10 \%$ des taxes au district. Un CED a extrait 80 tonnes de déchets, puis a mis en place un système de collecte, de tri sélectif et de revente des déchets qui emploie 46 personnes. Par ailleurs, le CED a mis en place une procédure d'enregistrement des prestataires de service (par exemple les loueurs de mules), afin d'appliquer une éco-taxe. Ceci lui a permis, avec l'aide de la réserve, de mettre en place différents services et de créer un centre d'interprétation, source d'emplois qualifiés. Le CED est ainsi devenu un acteur clé du développement local et de la conservation de la nature. Une dynamique latente a été activée. On raisonnera donc tant en terme de création que de réarrangements institutionnels.

\subsection{Questions d'échelles : le temps et les espaces du dialogue}

Dans quelle échelle temporelle s'inscrit-on? L'observation de temps forts de dialogue, au sein d'instances de participation, conduit à constater que plus le dialogue est ponctuel, plus l'obligation de résultat pèse sur la qualité de ce dernier. Par exemple au Cambodge, une réunion entre environnementalistes et pêcheurs, accusés d'être responsables de la mortalité d'oiseaux du fait d'installations de pêche dépassant au-dessus de l'eau, se solde par un accord sur des actions de sensibilisation dont tous savent qu'elles seront inefficaces. C'est la figure de l'accord fictif (Figure 1), qui est d'autant plus problématique qu'il clôt la discussion. Le désaccord est préférable à un faux accord car il permet de relancer le processus de dialogue plus facilement. Il est indispensable de se donner le temps nécessaire à la conduite de véritables processus d'interconnaissance et de construction collective de solutions, afin d'éviter l'écueil que constitue l'accord fictif, observé à plusieurs reprises dans des instances convoquées de façon ponctuelle.

FIgURE 1. LES PRODUITS DU DIALOgUE : DÉSACCORD, ACCORD fICTIF, ACCORD CONSOLIDÉ

\begin{tabular}{|c|c|c|c|}
\hline & $\begin{array}{c}\text { Désaccord : } \\
\text { certains disent } \\
\text { non }\end{array}$ & $\begin{array}{l}\text { Consensus passif : } \\
\text { certains ne disent } \\
\text { ni oui, ni non }\end{array}$ & $\begin{array}{l}\text { Consensus actif : } \\
\text { tous disent oui à } \\
\text { l'accord proposé } \\
\text { ou construit } \\
\text { ensemble }\end{array}$ \\
\hline $\begin{array}{c}\text { Tous savent que la } \\
\text { solution n'est pas } \\
\text { efficace }\end{array}$ & \multirow{3}{*}{$\begin{array}{l}\text { Désaccord } \\
\text { explicite : il } \\
\text { permet de } \\
\text { relancer la } \\
\text { recherche d'un } \\
\text { accord }\end{array}$} & \multirow{2}{*}{\multicolumn{2}{|c|}{$\begin{array}{c}\text { Accord fictif } \\
\text { Zone du faux accord } \\
\text { lié à une obligation } \\
\text { de résultat }\end{array}$}} \\
\hline $\begin{array}{c}\text { Certains pensent que la } \\
\text { solution n'est pas } \\
\text { efficace }\end{array}$ & & & \\
\hline $\begin{array}{c}\text { Tous pensent que la } \\
\text { proposition est bonne, } \\
\text { que la solution est } \\
\text { efficace }\end{array}$ & & & Accord consolidé \\
\hline
\end{tabular}

En terme spatial, le dialogue est centré sur le territoire de la réserve, mais la participation du public n'est parfois possible que si l'on veille à établir un dialogue à d'autres niveaux. 
Par exemple au Bénin, les responsables de la réserve de la Pendjari soulignent le fait qu'ils ne peuvent engager un dialogue avec les acteurs locaux sans l'assentiment de leur hiérarchie et de certaines institutions publiques peu enclines à ouvrir l'action publique à la participation. Par ailleurs, ils ne peuvent agir sans coordination avec d'autres administrations qui disposent de prérogatives dans certaines zones. La participation du public est donc liée à plusieurs dimensions du dialogue. C'est d'abord le dialogue entre l'autorité de gestion et son autorité de tutelle, la mise en place de mécanismes de participation du public étant un acte significatif qui revient à reconnaitre les acteurs locaux légitimes pour exprimer leur point de vue, faire des propositions, voire contester l'action publique. C'est ensuite le dialogue entre les intervenants institutionnels. Par exemple dans le Nanda Devi, en Inde, les prérogatives de la réserve sont limitées aux espaces naturels alors qu'au Cambodge, sur le Tonle Sap, elles sont limitées par celles du ministère des pêches, qui gère la ressource halieutique. La participation du public n'a pas de sens en l'absence d'un dialogue entre ces intervenants. Ceci revêt tant d'importance que lorsqu'il s'agit d'identifier les «stakeholders", les responsables de la réserve citent une série d'administrations publiques entre lesquelles une coordination est recherchée. Enfin, des mécanismes de dialogue existent aussi entre acteurs locaux, indépendamment de la réserve : la participation du public interagit nécessairement avec ces mécanismes de coordination, qu'elle doit considérer.

\subsection{L'entrée en dialogue : pour la participation de tous}

En amont du dialogue se pose souvent le problème de la participation d'acteurs dotés d'un capital social et économique tel qu'ils n'ont pas intérêt à dialoguer, le rapport de force leur étant très favorable. La question est alors de savoir comment créer un intérêt au dialogue. Les réserves de biosphère du Lac Saint Pierre et de Manicouagan, au Canada, ont réussi à entrer en dialogue avec des groupes industriels très puissants en exploitant le cadre légal existant, les incitations marchandes auxquelles les entreprises sont sensibles, un large réseau de partenariats qu'elles se sont attachées à construire et en préférant une logique de coopération à une logique d'opposition vouée à l'échec. Au Canada, les industries forestières sont obligées d'ouvrir un espace de concertation pour élaborer un Plan d'Aménagement Forestier Durable et accéder à des certifications de leurs produits qui leur donnent une position favorable sur le marché. La réserve de Manicouagan a utilisé son réseau de partenaires pour être admis dans cet espace et y introduire des préoccupations qui lui sont propres, telles que la gestion du paysage. Il a proposé d'aider à l'élaboration du plan et s'est positionné comme éco-conseiller. Sur le Lac Saint Pierre, les acteurs de la réserve ont utilisé un film montrant l'impact de la vitesse des navires sur l'érosion des berges, pour intéresser les armateurs - soucieux de leur image - à un dialogue. Dans tous ces cas, après avoir trouvé une "porte d'entrée ", les acteurs de la conservation de la nature proposent une offre de coopération. Les cas observés montrent l'intérêt de cette façon d'agir pour obtenir la participation de ces acteurs.

Pour les autres acteurs, comment obtenir leur participation ? La réunion est souvent le seul format de dialogue mobilisé, et ceci de façon très standard. Mais comment rendre le dialogue plus attractif, effectif et créatif? Certaines réserves de biosphère montrent la voie en jouant sur la convivialité d'une part, la projection de représentations simples de la réalité d'autre part. A Manicouagan, la réserve s'appuie sur un schéma très simple sur lequel figurent les cinq ressources majeures du territoire, végétales, fauniques, 
paysagères, minérales et hydriques, disposées sur un triangle dont les sommets sont des pôles environnemental, économique et social. Ce schéma est un support de dialogue qui permet d'introduire beaucoup d'éléments : les acteurs car ceux-ci peuvent être associés à chaque ressource; des objectifs avec deux ressources (le paysage et la faune) dont la gestion devrait conduire à moduler ce qui se fait dans l'exploitation des trois ressources soumises à des usages industriels; l'actualité car tous les problèmes qui surgissent peuvent être situés dans ce schéma. La convivialité est un autre facteur très important pour mobiliser les participants et stimuler l'échange. La réserve de biosphère de South Nova, au Canada, voulait mettre en place des focus group au niveau local. Rencontrant peu de succès avec des réunions classiques, elle a créé des « groupes de tables de cuisine » où l'on se réunit entre voisins. Il n'y a pas d'agenda spécifique mais une question générale : comment améliorer la qualité de la vie dans notre territoire ? Leur forme a permis à ces kitchen table group d'avoir beaucoup de succès et de briser les barrières entre scientifiques et profanes. Enfin, certaines réserves introduisent une gamme de supports et outils pour le dialogue, tels que la cartographie participative des ressources, les échanges d'expériences, des interviews d'acteurs, des formations suivies ensemble, des rechercheaction, etc... Ces outils visent non seulement à échanger mais aussi à collecter des savoirs locaux et visualiser l'information de façon à la rendre partageable et discutable; à acquérir collectivement de nouvelles capacités de compréhension et de proposition; à stimuler la créativité. Ces objectifs sont complémentaires, les outils mobilisés pour les atteindre le sont aussi, d'où l'intérêt de combiner des outils diversifiés et de sortir d'un format d'échange unique et standard.

\section{Conclusion}

L'analyse comparative ici présentée a été réalisée dans des contextes très divers. Force est de constater que la participation du public pose des problèmes différents d'un pays à l'autre, en fonction de plusieurs facteurs contextuels. Il est d'abord plus difficile de créer un îlot de participation au sein d'une vie et d'une action publique auxquelles le public n'est pas associé, que là où existe déjà une trame dense d'espaces de dialogue. D'autres facteurs déterminent des situations distinctes : la valeur accordée par les pouvoirs publics à la parole des citoyens diffère implicitement d'un pays à l'autre ; l'histoire des rapports entre les citoyens et l'Etat détermine la confiance qui existe au départ ; la façon dont sont considérés les droits de propriété détermine ce qui est discutable et ce qui ne l'est pas ; les modalités d'expression ou de non expression des conflits dépendent de facteurs culturels ou politiques. Face à la diversité des contextes, il ne s'agit pas de proposer une méthode mais des points de repères : l'analyse des formes de participation et des paramètres des espaces de dialogue mis en place nous a permis d'en proposer certains. En termes de recherche, cette diversité complique l'analyse mais constitue un atout majeur pour identifier des récurrences dans les facteurs qui facilitent ou entravent une participation constructive, de telles récurrences apparaissant plus clairement dans des contextes hétérogènes que lorsque l'analyse comparative est réalisée dans des contextes similaires.

Les réserves de biosphère, avec le même dispositif de base présent dans de nombreux pays et contextes, constituent un support intéressant pour une telle analyse. Le cas des réserves de biosphère montre ici que la participation du public épouse des contours très différents, selon qu'il s'agit d'une participation contributive, d'une participation limitée à des opérations d'information ou de consultation, ou d'une véritable concertation. Mais 
au-delà de la participation du public aux actions et instances de dialogue proposées par le gestionnaire de la réserve, on observe que certains préfèrent se mettre en retrait et semer des graines de dialogue et d'initiative dans le territoire qu'ils veulent gérer. Ils accompagnent ensuite des initiatives qu'ils ne maîtrisent pas mais qui assurent la participation du public et sa contribution aux objectifs de la réserve. Il s'agit alors de créer un milieu dialoguant et actif autour de la conservation de la nature. Ceci se rapproche de l'auto-mobilisation identifiée par Pretty (1995) comme une forme de participation, mais cette mobilisation est stimulée par l'autorité en charge de la gestion de l'environnement. Notre analyse nous conduit finalement à proposer une typologie des voies et moyens de la participation du public à la conservation de la nature et au développement durable : elle s'inspire de celle de Pretty (1995) et plus secondairement de celle d'Arnstein (1969), mais apporte des précisions et explore un champ des possibles toujours plus vaste.

Une limite majeure de cette recherche est que nous n'avons pu aborder la question de l'évaluation des résultats des dispositifs de participation, en termes de développement et de conservation, faute d'indicateurs disponibles et homogènes d'une réserve à l'autre. La réserve Maya, au Guatemala, a conçu un jeu d'indicateurs qu'il serait intéressant de tester dans plusieurs réserves pour engager une évaluation comparative. Ceci aurait été d'autant plus intéressant que le principe même de la participation du public à la conservation de l'environnement est aujourd'hui mis en cause. Raymond (2009) et Ballan (2011) s'interrogent ainsi sur une idéalisation de la participation des citoyens concernant l'environnement. La Branche (2009) précise également que la participation n'est pas suffisante en soi pour garantir une prise en compte du développement durable et de l'environnement, puis précise qu'il existe très peu d'études empiriques portant sur l'impact environnemental des procédures de participation. Nous avons analysé la façon dont diverses voies sont explorées dans les réserves de biosphère pour rendre effective la participation du public : évaluer l'opportunité de la participation et comparer l'intérêt des différentes modalités envisagées, en termes d'environnement et de développement durable, constitue une piste pour prolonger ces recherches.

\section{BIBLIOGRAPHIE}

Arnstein, S.R., 1969, « A Ladder of Citizen Participation », Journal of the American Planning Association, Vol. 35, n4 July, p. 216-224.

Atger C., Gallety J.C., Rousset M., 2000, La concertation en aménagement : éléments méthodologiques, Ministère de l'Equipement, des transports et du logement, Paris, Editions Tec \& Doc.

Ballan E., 2011, « Démocratie et environnement, un mariage arrangé », Revue Territoires, n516.

Beierle T.C. 1999, « Using social goals to evaluate public participation in environmental decisions ", Review of Policy Research, Vol. 16, p. 75-103.

Bertrand N., Moquay P., 2004, «La gouvernance locale, un retour à la proximité », Economie Rurale , n²80, p.77-95. 
Beuret J.E., 2006a, La conduite de la concertation, pour la gestion de l'environnement et le partage des ressources, Paris, L'Harmattan.

Beuret J.E., 2006b. « Dialogue and concertation in biosphere reserves: issues and challenges », in: Bouamrane, M. (Ed.), Biodiversity and Stakeholders: concertation itineraries, Note technique 1-2006, Paris, UNESCO, p. 8-21.

Beuret J.E., 2006c, Environnement et développement mis en dialogue dans les réserves de biosphère : rapport technique, UNESCO, Paris, 93 p. + fiches techniques + annexes.

Beuret J.E., Cadoret A., 2010, Gérer ensemble les territoires : vers une démocratie coopérative, Paris, Editions Charles Leopold Mayer.

Bouamrane M., (Ed.), 2006, Biodiversité et acteurs : des itinéraires de concertation. La contribution des réserves de biosphère, Note technique 1-2006, Paris, UNESCO Ed.

Callon M., Lascoumes P., Barthe Y., 2001, Agir dans un monde incertain : essai sur la démocratie technique, Paris, Seuil.

Camagni R., Maillat D., 2006, Milieux innovateurs, théorie et politique, Paris, Economica.

De Rosnay, J., 1975, Le macroscope : vers une vision globale, Paris, Seuil.

Coppin O., 2002, «Le milieu innovateur : une approche par le système », Innovations, 2002/2, $\mathrm{n}^{\circ} 16$, p. 29-50.

Favereau O., 1989, « Marchés internes, marchés externes », Revue Economique, 2/1989, pp. 273-328.

Fung A., 2003, « Recipes for Public Sphere: Eight Institutional Design Choices and Their Consequences », The Journal of Political Philosophy, Vol. 11, n 3, p. 338-367.

Fung A., 2006, « Varieties of Participation in Complex Governance », Public Administration Review, December, p. 66-75.

Habermas J., 1978, Raison et légitimité : problèmes de légitimation dans le capitalisme avancé, Paris, Payot.

Jean B., Ependa Muteba Wa A., 2004, «Le capital social et le renforcement des « capacités de développement » des communautés rurales : les enseignements d'une étude canadienne », Revue d'Economie Régionale et Urbaine, n5, p. 673-694.

La Branche S., 2009, «L'insoutenable légèreté environnementale de la participation : une problématisation », VertigO, Vol. 9, n¹, mai, http://vertigo.revues.org/8346

Laganier R., Villalba B., Zuindeau B., 2002, « Le développement durable face au territoire : éléments pour une recherche pluridisciplinaire », Développement Durable et Territoires, dossier 1, http://developpementdurable.revues.org/774

Maikhuri R.K., Rao K.S., 2006, Assessment and promotion of eco-tourism in Nanda Devi Biosphere Reserve : an option to resolve policy-people conflict, Final Progress report, G.B.Pant Institute of Himalayan Environment and Development, New Dehli.

Pretty J.N., 1994, « Alternative Systems of Inquiry for a Sustainable Agriculture », IDS Bulletin, Vol. $25, n^{\circ} 2$, p. 39-48.

Pretty J.N., 1995, « Participatory Learning For Sustainable Agriculture », World Development, Vol. $23, n^{\circ} 8$, p. $1247-1263$

Raymond R., 2009, « La "société civile", ce "nouvel" acteur de l'aménagement des territoires », L’information géographique, Vol. 73, n², p. 10-28. 
Rowe G., Frewer L.J., 2005, « A Typology of Public Engagement Mechanisms », Science, Technology, \& Human Values, Vol. 30, n² 2, p. 251-290.

Trom D., 2003, «Introduction », in : Laborier P. et D. Trom (dir.), Historicités de l'action publique, Paris, PUF.

Touzard H., 2006, « Consultation, concertation, négociation : une courte note théorique », Négociations, Vol ; 5, 2006/1, p. 69-74.

\section{NOTES}

1. Ce projet de recherche a bénéficié du soutien d'Agrocampus Ouest et du programme Man And Biosphere de l'UNESCO. Mais les propos tenus dans cet article n'engagent que leur auteur.

\section{RÉSUMÉS}

La participation du public à la conservation de la nature emprunte des voies très diverses, comme le montre l'analyse comparative de douze réserves de biosphère, menée dans dix pays. Il peut s'agir d'une participation contributive, d'une participation limitée à des opérations d'information ou de consultation, d'une véritable concertation ou de mécanismes par lesquels le gestionnaire de la réserve tente de stimuler l'émergence d'initiatives décentralisées et de créer un milieu dialoguant et innovant autour de la conservation de la nature. Après la présentation du cadre conceptuel et de la démarche d'analyse, cet article expose la gamme des dispositifs mis en œuvre pour assurer la participation du public, puis aborde les questions-clés posées par la mise en dialogue de l'environnement et du développement, dans une perspective de développement durable.

The general public can help to conserve nature in many different ways, as shown by the comparative analysis of twelve biosphere reserves carried out in ten countries. This may be in the form of a contributive participation, a participation limited to information or consultation operations, a real negotiation or measures by which managers of biosphere reserves try to encourage independent initiatives and create innovative settings for discussion on nature conservation. This article presents the conceptual framework and the analytical approach and then describes the range of schemes set up to allow the participation of the public. It then looks at the key questions to be answered when setting up discussions between environment and development, in a sustainable development perspective.

\section{INDEX}

Keywords : biodiversity, biosphere reserves, comparative analysis, governance, participation Mots-clés : analyse comparative, biodiversité, gouvernance, participation, réserves de biosphère 


\section{AUTEUR}

\section{JEAN-EUDES BEURET}

Jean-Eudes Beuret est agro-économiste, professeur au laboratoire Systèmes de Production et Développement Rural d'Agrocampus-Ouest. Il s'intéresse à la gouvernance et à la conduite de processus de concertation dans des situations très diverses, qu'il s'agisse de partager des ressources naturelles, d'élaborer des projets dans les territoires, de gérer des espaces naturels protégés, de développer la démocratie participative, en Europe ou dans les pays du Sud. Il a notamment publié La conduite de la concertation pour la gestion de l'environnement et le partage des ressources, aux Editions l'Harmattan et, avec Anne Cadoret Ensemble pour gérer les territoires : vers une démocratie coopérative, aux Editions Charles Leopold Mayer. Mail : beuret@agrocampusouest.fr 